\title{
Plasmodium Infection Suppresses Colon Cancer Growth by Inhibiting Proliferation and Promoting Apoptosis Associated with Disrupting Mitochondrial Biogenesis and Mitophagy in Mice
}

Xin Yao ( $\nabla$ monicayx18@163.com )

Bengbu Medical College https://orcid.org/0000-0002-9279-8966

Yujie Cao

Bengbu Medical College

Li Lu

Bengbu Medical College

Yuanxia Xu

Bengbu Medical College

Hao Chen

Bengbu Medical College

Chuanqi Liu

Bengbu Medical College

Dianyi Chen

Bengbu Medical College

Kexue Wang

Bengbu Medical College

Jingxiang $\mathbf{X u}$

Bengbu Medical College

Runqi Fang

Bengbu Medical College

Hui Xia

Bengbu Medical College

Jiangyan Li

The First Affiliated Hospital of Bengbu Medical College

Zhiyong Tao

Bengbu Medical College

Qiang Fang

Bengbu Medical College 


\section{Research Article}

Keywords: Plasmodium, Colon cancer, Mitochondrial apoptosis, Mitochondrial biogenesis, Mitophagy

Posted Date: January 11th, 2022

DOI: https://doi.org/10.21203/rs.3.rs-1224779/v1

License: (c) (i) This work is licensed under a Creative Commons Attribution 4.0 International License. Read Full License 


\section{Abstract}

Background: Colon cancer is a common gastrointestinal tumor with a poor prognosis, which makes it urgent to explore new therapeutic strategies. The anti-tumor effect of Plasmodium infection has been reported in some murine models, but it is not clear whether it has an anti-colon cancer effect. In this study, we investigated the anti-colon cancer effect of Plasmodium infection and its related mechanisms using a mouse model of colon cancer.

Methods: An experimental model was established by intraperitoneal injection of Plasmodium yoeliiinfected erythrocytes into mice with colon cancer. The size of tumors was observed dynamically in mice, and the expression of Ki67 detected by immunohistochemistry was to analyze tumor cells proliferation. Apoptosis was assessed by Terminal deoxynucleotidyl transferase (TdT) dUTP Nick-End Labeling (TUNEL) staining, and the expression of apoptosis concerned proteins, including Bax, Bcl-2, Caspase-9, Cleaved Caspase-3, were detected by western blot and immunohistochemistry, respectively. Transmission electron microscopy (TEM) was used to observe the ultrastructural change of colon cancer cells. And the expression of mitochondrial biogenesis correlative central protein, PGC-1a, and mitophagy relevant crucial proteins, PINK1/Parkin, were detected by western blot.

Results: We found that Plasmodium infection reduced the weights and sizes of tumors and decreased the expression of Ki67 in colon cancer-bearing mice. Furthermore, Plasmodium infection promoted mitochondria-mediated apoptosis in colon cancer cells, as evidenced by the increased proportion of TUNEL-positive cells, the up-regulated expression of Bax, Caspase-9, and Cleaved Caspase-3 proteins, and the down-regulated expression of $\mathrm{Bcl}-2$ protein. In colon cancer cells, we found destroyed nucleus, swollen mitochondria, missing cristae, and the decreased number of autolysosomes. In addition, Plasmodium infection disturbed mitochondrial biogenesis and mitophagy through the reduced expression of PGC-1a, PINK1, and Parkin proteins in colon cancer tissues.

Conclusions: Plasmodium infection can play an anti-colon cancer role in mice by inhibiting proliferation and promoting mitochondria-mediated apoptosis in colon cancer cells, which may relate to mitochondrial biogenesis and mitophagy.

\section{Background}

Colon cancer is one of the most frequent digestive system tumors, ranking fifth in new cases and fatalities among all malignancies worldwide [1]. At present, although surgical resection, radiotherapy, and conventional chemotherapy are used routinely in the treatment of colon cancer [2], the therapeutic effect is still not satisfactory, and it is urgent to explore new therapeutic strategies.

As early as the beginning of the last century, Plasmodium, one of the most important parasites, has been tried to treat diseases such as advanced syphilis [3]. Since then, the relationship between Plasmodium and cancer has also received some attention. According to the report, there was an inverse correlation between the incidence of malaria and the mortality rate of some cancer from 1955 to 2008 globally[4]. 
The antitumor effect of Plasmodium infection has been reported in some animal studies, including lung cancer [5], hepatocellular carcinoma [6], leukaemia [7], and melanoma [8]. However, it has not been reported whether Plasmodium infection could inhibit colon cancer.

In the reported studies, the antitumor mechanism of Plasmodium infection focused on host antitumor immune response [5], inhibiting tumor microenvironment signals [9], and anti-tumor angiogenesis [10]. However, what happens to tumor cells doesn't get enough attention. It is well known that the infinite proliferation ability and loss of apoptosis ability of tumor cells are the key to tumor growth [11]. Many therapies exert anticancer effects by promoting tumor cell apoptosis, especially mitochondria-mediated apoptosis $[12,13,14]$. Some parasites, such as Toxoplasma gondii [15], exerted an anti-tumor function by promoting apoptosis. However, it is not clear whether Plasmodium infection also plays an anti-tumor role by inhibiting proliferation and promoting apoptosis.

An increasing number of studies have shown that there is a close link between mitochondria and cancer development $[16,17]$. Mitochondria is fundamental for cell growth and proliferation in cancer, and metabolic imbalances or increased resistance to mitochondrial apoptosis are prominent features for cancer cell [18]. Indeed, mitochondria maintain cellular homeostasis by regulating mitochondrial biogenesis and mitophagy $[19,20]$. Mitochondrial biogenesis can generate new functional mitochondria to increase the number of mitochondria through transcriptional level regulation [21], and inhibition of mitochondrial biogenesis can inhibit tumor cell proliferation [22]. Mitophagy is a form of autophagy by which the damaged or superfluous mitochondria are phagocytosed and degraded [23,24,25], and inhibition of mitophagy can aggravate mitochondrial damage and promote mitochondria-mediated apoptosis $[26,27]$. Because mitochondria play a key role in cell proliferation and death, plenty of studies have proved that targeting mitochondria could restrain tumor growth or promote apoptosis [28, 29]. However, whether mitochondrial biogenesis and mitophagy are involved in the anti-tumor effect of Plasmodium infection requires further exploration.

In this study, we used a murine colon cancer model to investigate the anti-colon cancer effect of Plasmodium yoelii infection and its potential mechanism. Our results suggested that Plasmodium infection could inhibit tumor growth in mice by suppressing proliferation and promoting apoptosis, which might relate to the inhibition of mitochondrial biogenesis and mitophagy in colon cancer cells. Those results will contribute to developing novel strategies for colon cancer treatment.

\section{Methods}

Source of animals, cells, and parasites

BALB/c mouse, which featured females, 6-8 weeks old and 18-20 g, were purchased from Chang Zhou Cavens Laboratory Animal Ltd and maintained under a controlled temperature of $20-25{ }^{\circ} \mathrm{C}$, relative humidity of $40-50 \%$. All experiments were reviewed and approved by the Experimental Animal Management and Ethics Committee of Bengbu Medical College, Bengbu, China (approval no: 2021-322). 
The nonlethal Plasmodium yoelii 17XNL ( $P$. yoelii 17XNL) strain was donated by Professor Yaming Cao of China Medical University and preserved by our laboratory.

The CT26.WT cell, mouse colon cancer cell lines, were purchased from Guangzhou Cellcook Biotechnology Company and cultured in Roswell Park Memorial Institute (RPMI) 1640 medium (Gibco, USA) containing $10 \%$ fetal bovine serum (FBS) and $1 \%$ penicillin-streptomycin (100 units $/ \mathrm{ml}$ penicillin and $100 \mu \mathrm{g} / \mathrm{ml}$ streptomycin) under $37{ }^{\circ} \mathrm{C}$ and $5 \% \mathrm{CO}_{2}$ in an incubator.

Establishment of the murine colon cancer model and infection with the $P$. yoelii

A colon cancer model was established as described Masayoshi [30]. As the subcutaneous model of colon cancer-bearing mice, ten BALB/c mice were established by subcutaneous inoculation of $5 \times 10^{6}$ CT26.WT cells below the axilla of the right forelimb in the mouse. From the date of tumor formation (six days after tumor cell injection), ten mice were divided into two groups randomly (five animals per group). CT26.WT +P.y as the experimental group, each mouse was intraperitoneally injected with $1 \times 10^{6} \mathrm{P}$. yoelii infected erythrocytes. The control group (CT26.WT) of the mouse was intraperitoneally injected with $1 \times 10^{6} P$. yoelii uninfected erythrocytes. The tumor growth was observed every two days from the date of tumor emergence.

The dynamical observation of tumor growth

When the tumor was measurable in tumor-bearing mice (on the sixth day after CT26.WT cells inoculation), the long diameter $a(\mathrm{~mm})$ and short diameter $b(\mathrm{~mm})$ of the tumor was measured with vernier caliper every two days, and the tumor volume was calculated according to the formula, $V=\left(a b^{2}\right) / 2$. On the 18th day after inoculating Plasmodium-infected erythrocytes (on the 24th day after modeling), the tumor-bearing mice were sacrificed to harvest tumors, weigh, and photograph for further analysis.

Immunohistochemistry staining

The tumor specimens were fixed with $4 \%$ paraformaldehyde solution for paraffin embedding and sectioning. After xylene dewaxing, gradient ethanol hydration, and antigen high-pressure repair, they were stained with primary antibody (Abcam, USA; Cell Signal Technology, USA;) (Ki67 antibody, 1:400; Bax antibody, 1:400; Bcl-2 antibody, 1:500; Caspase-9 antibody, 1:300; Caspase-3 antibody, 1:300; Cleaved Caspase-3 antibody, 1:2000), and incubated at $37^{\circ} \mathrm{C}$ for one hour. The second antibody was cultivated at $37^{\circ} \mathrm{C}$ for 30 minutes. After DAB coloration and observation under a high magnification microscope $(\times 400)$, the positive results were expressed in the brown position. Image-Pro Plus 6.0 software was used for statistical analysis, and average optical density value was used to reflect protein expression level.

TUNEL staining

The tumor tissue specimens were fixed with $4 \%$ paraformaldehyde and sectionalized with paraffin embedding. Terminal deoxynucleotidyl transferase (TdT) dUTP Nick-End Labeling (TUNEL) assay 
performed using the One-Step TUNEL Apoptosis Assay Kit, DAPI Staining Solution, and Antifade Mounting Medium (Beyotime Biotechnology), according to the manufacturer's protocol. Under a fluorescence microscope $(\times 400)$, the normal nucleus is blue and the apoptotic nucleus is green. All images were acquired using the Nikon Eclipse 50i microscope system and Image-pro Plus 6.0 software with standard image processing. The apoptosis rate was calculated by the number of TUNEL positive nuclei/number of DAPI stained nuclei.

Western blot

RIPA buffer (Beyotime Biotechnology, China) was used to extract total protein from the tumor tissue, and the BCA method was used for quantitative detection of protein. The proteins were isolated in $12 \%$ sodium dodecyl sulfate-polyacrylamide gel electrophoresis (SDS-PAGE gel) and transferred onto the PVDF membrane. After being sealed with $5 \%$ defatted milk at room temperature, the PVDF membrane was incubated with the specific primary antibodies against PGC-1a, PINK1, Parkin (ABclonal) (rabbit polyclonal antibody, 1:1000), Bax, Bcl-2, Caspase-9, Caspase-3, Cleaved Caspase-3 (Cell Signal Technology, USA) (rabbit anti-mouse monoclonal antibody, 1:1000), at $4{ }^{\circ} \mathrm{C}$ overnight. After being incubated with the second antibody (Cell Signal Technology, USA) (HRP-conjugated goat anti-rabbit IgG, 1:2000) was added and incubated at room temperature for an hour, the protein bands were visualized by enhanced chemiluminescence reagent (Merck Millipore, USA) and detected using Biolmageing Systems (BIO-RAD ChemiDoc ${ }^{T M}$ MP Imaging System, USA). The results were quantified by Image-J software. The relative protein expression was expressed by the gray value of target protein /GAPDH gray value.

Morphological observation by transmission electron microscopy (TEM)

The tumor tissue samples were harvested and fixed at $4{ }^{\circ} \mathrm{C}$ for $2-4$ hours with glutaraldehyde. Then they were fixed at room temperature $\left(20^{\circ} \mathrm{C}\right)$ for $2 \mathrm{~h}$ with $1 \%$ osmium and $0.1 \mathrm{M}$ phosphoric acid buffer $\mathrm{PB}$, rinsed with $0.1 \mathrm{M}$ phosphoric acid buffer PB. After dehydration in graded ethanol and acetone, the samples were embedded Epon 812. The ultrathin sections were stained with uranium acid and lead uranium and then observed under TEM (Hitachi, Japan).

\section{Statistical analysis}

For parameter data in each experiment, the unpaired two-tailed Student's t-test was used to analyze the differences between groups. Statistical analyses were performed with GraphPad Prism software (version8). All experiments were repeated more than three times, and the data were expressed as the mean \pm standard error of the mean (SEM). A $P$-value less than 0.05 was considered statistically significant.

\section{Results}

Inhibition of colon cancer growth by Plasmodium infection in mice 
The mouse model of colon cancer was established by subcutaneous inoculation. On the sixth day after tumor cells inoculation, erythrocytes infected with $P$. yoelii were injected intraperitoneally into tumor-being mice (Fig. 1b). At first, there was no significant difference in tumor size between the Plasmodium infection group $\left(5.905 \pm 1.357 \mathrm{~mm}^{3}\right)$ and the control group $\left(3.487 \pm 0.758 \mathrm{~mm}^{3}\right)$ on day six (Fig. 1a). From the 15th day of tumor cells inoculation, tumor size in the $P$. yoelii-infected group was not only significantly reduced compared to the un-infection group, but tumor growth was remarkably slow in the CT26.WT+P.y group. On the 18th day after being inoculated with Plasmodium-infected erythrocytes, the tumors were strikingly smaller in size and weight than the control group (Fig. 1b, c). The results indicated that Plasmodium infection could inhibit the growth of colon cancer in mice.

Plasmodium Infection suppressed the proliferation of colon cancer cells in mice

To determine the effect of Plasmodium infection on the proliferation of colon cancer cells in mice, we examined the expression of Ki67, a cell proliferation marker, using immunohistochemistry staining. We found that the expression of Ki67 was decreased obviously in the P. yoelii infection group than the control group (Fig. 2a). There were statistically significant differences in the percentage of Ki67 positive cells between the two groups (Fig. 2b). It was suggested that Plasmodium infection could suppress the proliferation of colon cancer cells in vivo.

Apoptosis of colon cancer cells induced by Plasmodium infection in mice

Apoptosis of tumor cells was detected by TUNEL assay, in which enhanced green fluorescence represented the TUNEL positive cells. A small number of apoptotic cells were found in the non-infected Plasmodium group (CT26.WT), while a large number of TUNEL positive cells were detected in the infected Plasmodium group (CT26.WT+P.y) (Fig. 3a). The CT26.WT+P.y group displayed a variation in the proportion of cell apoptosis compared with the CT26.WT group (Fig. 3b). It indicated that Plasmodium infection could accelerate the apoptosis of colon cancer cells.

The mitochondrial apoptosis activation by Plasmodium infection

To further explore the mechanism of Plasmodium infection-induced apoptosis, we used western blot to detect the expression of apoptotic proteins in the mitochondrial pathway. We observed that the expression levels of pro-apoptotic proteins containing Bax, Caspase-9, and Cleaved Caspase-3 were upregulated, while the expression of anti-apoptotic protein Bcl-2 was down-regulated after Plasmodium infection compared with the control group (Fig. 4a, b).

In addition, the effects of Plasmodium infection on the expression of mitochondrion-mediated apoptosis proteins were observed through immunohistochemistry staining (Fig. 4c, d). Consistent with the results of western blot, we found that the expression of Bax, Caspase-9, and Cleaved Caspase- 3 in the P. yoeliitreated model was increased noticeably. Nevertheless, there were less brown areas of Bcl-2 expression in the CT26.WT+P.y group than the CT26.WT group. These results suggested that Plasmodium infection 
involved in regulating the expression of apoptotic proteins in the mitochondrial pathway leading to mitochondrion-mediated apoptosis.

The mitochondria and nucleus of colon cancer cells changed by Plasmodium infection in tumor-being mice

As shown in Fig. 5, we used TEM to further observe changes in the ultrastructure of colon cancer cells among the two groups. After Plasmodium treatment, colon cancer cells displayed severe edema, nuclear atypia, chromatin clumpy agglutination, and nuclear disintegration. Mitochondria were severely swollen and enlarged, the matrix in the membrane dissolved, and the cristae disappeared and vacuolated in the CT26.WT+P.y group compared to the control group. Moreover, under TEM, we could see only one autolysosome in the $P$. yoelii-treated group in this field, whereas there were three autolysosomes in the control group. The results revealed that Plasmodium infection-induced tumor cell damage was associated with mitochondrial and nuclear damage in colon cancer cells.

Plasmodium infection disrupted the mitochondrial biogenesis in the colon cancer model

To assess the effect of parasite infection on mitochondrial biogenesis, we used western blot to analyze a key regulator of mitochondrial biogenesis, PGC-1a. And the result showed that the expression of PGC-1a protein in the parasite-treated group was reduced evidently in contrast to the control group (Fig. 6). It was demonstrated that Plasmodium infection could disrupt mitochondrial biogenesis in colon cancer.

Plasmodium infection attenuated mitophagy in colon cancer-bearing mice

PINK1/Parkin-mediated pathway is the most important pathway of mitophagy, which is crucial for maintaining mitochondrial function and integrity [31]. To evaluate the effect of Plasmodium infection on mitophagy in colon cancer, we, therefore, examined the expression of mitophagy-related proteins. Western blot results showed that the levels of PINK1 and Parkin were declined after Plasmodium infection (Fig. 7). The results expressed that Plasmodium infection could attenuated mitophagy leading to mitochondrial dysfunction in colon cancer.

\section{Discussion}

As one of the most ordinary malignant tumors, colon cancer does pose an extreme threat to human health [32]. Due to the high incidence and mortality of colon cancer, researchers who have devoted themselves to reducing mortality and improving the quality of life for patients have been seeking new therapeutic methods to inhibit the growth, recurrence, and metastasis of colon cancer [33]. Previously, researchers and our team all have found that Plasmodium infection had antitumor effects on some cancer in some mouse models, but it is not clear whether it has similar influences on colon cancer yet. Therefore, in this study, we investigated the anti-colon cancer effect of Plasmodium infection in mice. 
In this study, we have found that Plasmodium infection decelerated the growth of tumors and reduced the size and weight of the tumors in the murine colon cancer model. These results indicated that Plasmodium infection could play an anti-colon cancer role in mice.

Infinite proliferation is one of the important characteristics of tumor cells [34]. Inhibition of cell proliferation could effectively inhibit tumor growth [35]. Ki67 protein, a nuclear antigen related to cell proliferation, is expressed in G1, G2, S, and M period, but no expression in the G0 period [36]. As one of the most reliable indicators for detecting cell proliferation activity of tumor cells, the function of Ki67 protein is closely associated with the process of cell mitosis [37, 38]. In this study, the expression of Ki67 in colon cancer tissues was distinctly decreased after parasite treatment, suggesting that Plasmodium infection could suppress the proliferation of colon cancer cells and further inhibit tumor growth in tumorbearing mice.

Apoptosis, the most common form of programmed cell death, is one of the keys to maintaining healthy cell homeostasis [39]. Apoptosis resistance is another crucial characteristic of tumor cells [40]. As an important method for prevention and treatment, induction of apoptosis is widely used in antitumor drug research [41]. There are three pathways of apoptosis in mammalian cells, containing the Bcl-2 regulatory mitochondrial pathway, the death receptor pathway, and the endoplasmic reticulum pathway $[42,43,44]$. Mitochondrial apoptosis is the primary form of apoptosis [45]. When mitochondria are damaged, Bax is transported into mitochondria to initiate apoptosis, triggering Cytochrome $\mathrm{C}$ releasing in the mitochondria, which further activates the Caspase cascade resulted in apoptosis $[46,47,48]$.

In the tumor-being mice infected with $P$. yoelii, we found the increased proportion of TUNEL-positive cells, the up-regulated expression of pro-apoptotic factors Bax, Caspase-9, and Cleaved Caspase-3, and the down-regulated expression of anti-apoptotic factor Bcl-2 in colon cancer tissues, which indicated Plasmodium infection promoted mitochondria-mediated apoptosis. Since inadequate apoptosis is one of the typical characteristics of cancer, promoting apoptosis will lead to the death of cancer cell. Thus, we have reason to believe that Plasmodium infection promoting mitochondria-mediated apoptosis could inhibit tumor growth through promoting mitochondria-mediated apoptosis and play an anti-colon cancer role in tumor-bearing mice like some anti-tumor drugs $[49,50]$.

Mitochondrion, a subcellular organelle, which plays a major role in cell energy control and metabolism, is essential for the survival and growth of cells [51, 52]. As regulators involved in the pathways of mitochondrial biogenesis, mitophagy, carcinogenesis, and tumor cell death, including mitochondriamediated apoptosis, mitochondria may be considered as potential therapeutic targets for cancer [53]. In this study, we found that the mitochondria and nucleus of colon cancer cells were damaged severely in the Plasmodium-treated mice, mainly with the mitochondrial cristae disappearance and vacuolation. The results implicated that the antitumor effect of Plasmodium infection may relate to mitochondria. The maintenance of mitochondrial homeostasis depends on the interaction between mitochondrial biogenesis and mitophagy [54]. PGC-1 a protein dominated mitochondrial biogenesis and protected tumor cells from apoptosis $[55,56]$. Those studies both in vivo and in vitro certified that down-regulated 
expression of PGC-1a induced apoptosis through the mitochondrial pathway [57]. Recent study has revealed a potential relationship between mitochondrial biogenesis and apoptosis [58]. In the early stage of apoptosis, mitochondria produced energy to sustain homeostasis, leading to enhanced mitochondrial biogenesis [59]. As the massive production of reactive oxygen species (ROS), mitochondrial energy metabolism was unbalanced, mitochondrial biogenesis was weakened, mitochondrial membrane potential was decreased, Cytochrome $\mathrm{C}$ was released, and the Caspase pathway was activated, leading to apoptosis $[60,61]$. In this study, we found that the expression of PGC-1 a protein in colon cancer cells was reduced after $P$. yoelii infection. The results suggested that Plasmodium infection could inhibit mitochondrial biogenesis in colon cancer cells. Inhibition of mitochondrial biogenesis could promote apoptosis and inhibit proliferation, which may be one of the mechanisms underlying the antitumor effects of Plasmodium infection.

Autophagy is a process in which intracellular components are degraded into autophagosomes and combined with the lysosomes to form autolysosomes, resulting in the degradation of their encapsulated contents $[62,63]$. More and more studies have demonstrated that autophagy is a self-protective mechanism for cells $[64,65]$. Mitophagy, a type of mitochondria-specific autophagy, is a self-protective process that dysfunctional mitochondria are degraded selectively [66]. When mitochondria are damaged, PINK1 protein hydrolysis is restrained and expressed steadily on the mitochondrial outer membrane. Then, PINK1 recruits Parkin to the outer membrane of mitochondria and ubiquitinates multiple mitochondrial outer membrane proteins to mediate mitophagy, eventually removing damaged mitochondria $[67,68]$. In the study, we found that the number of autolysosomes was decreased and the expression of PINK1/Parkin proteins were declined in colon cancer after Plasmodium infection. The results meant that Plasmodium infection could inhibit mitophagy in colon cancer-bearing mice leading to disruption of mitochondria and mitochondrial dysfunction. Many studies have shown that suppression of autophagy, including mitophagy, could accelerate apoptosis resulting in tumor cell death, ultimately inhibiting tumor growth $[69,70]$. Therefore, in colon cancer cells, Plasmodium infection could inhibit mitophagy, thus promoting apoptosis and ultimately inhibiting tumor growth, which might be one of the anti-colon cancer mechanisms of Plasmodium infection.

In this study, although we found that Plasmodium infection might inhibit proliferation and promote apoptosis by controlling mitochondrial biogenesis and mitophagy, it is not clear why Plasmodium infection could inhibit mitochondrial biogenesis and mitophagy. This suppression function may be connected with the subsequent effect of cytokines induced by immune effects after Plasmodium infection, the impact of components or metabolites of Plasmodium itself, or the result of changes in noncoding RNA expression caused by Plasmodium infection. The mechanism of Plasmodium infection controlling mitochondrial biogenesis and mitophagy remains to be further explored.

\section{Conclusions}

In this study, our findings firstly demonstrated that Plasmodium infection had significant anti-colon cancer effects in mice by inhibiting tumor cells proliferation and promoting mitochondrial apoptosis, 
which might relate to the inhibition of mitochondrial biogenesis and mitophagy (Fig. 8). The results presented here might inspire a new approach for the treatment of colon cancer.

\section{Abbreviations}

P. yoelii

Plasmodium yoelii

RPMI 1640

Roswell Park Memorial Institute 1640 medium

FBS

fetal bovine serum

TUNEL

Terminal deoxynucleotidyl transferase (TdT) dUTP Nick-End Labeling

TEM

transmission electron microscopy

SDS-PAGE gels

Sodium dodecyl sulfate-polyacrylamide gel electrophoresis

SEM

Standard error of the mean.

\section{Declarations}

\section{Acknowledgements}

We want to thank Professor Yaming Cao at China Medical University for providing $P$. yoelii 17XNL strain.

\section{Funding}

This work was supported by the Science Research Innovation Team Project of Anhui Colleges and Universities (No.2016-40), the 512 Talent Cultivation Program of Bengbu Medical College (No.by51201101), and the Postgraduate Scientific Research Innovation Program of Bengbu Medical College (No. Byycxz20005).

\section{Availability of data and materials}

The datasets supporting the findings of this article are included within the article and its additional file.

Authors' contributions

QF, ZT, JL, and XY conceived and designed the study. XY, YC, LL, YX, HC, CL, DC, KW, JX, and RF performed the experiments. $\mathrm{XY}, \mathrm{ZT}, \mathrm{HX}$, and QF analyzed the data. $\mathrm{XY}$ wrote the manuscript. QF critically revised the manuscript. All authors read and approved the final manuscript. 


\section{Ethics approval and consent to participate}

All animal experiments were reviewed and approved by the Experimental Animal Management and Ethics Committee of Bengbu Medical College, Bengbu, China (approval no. 2021-322).

\section{Consent for publication}

Not applicable.

\section{Competing interests}

The authors declare that they have no competing interests.

\section{Author details}

${ }^{1}$ Department of Microbiology and Parasitology, Bengbu Medical College, Bengbu, China.

${ }^{2}$ Anhui Key Laboratory of Infection and Immunity, Bengbu Medical College, Bengbu, China.

${ }^{3}$ School of Fundamental Sciences, Bengbu Medical College, Bengbu, China.

${ }^{4}$ Clinical Medical Department, Bengbu Medical College, Bengbu, China.

${ }^{5}$ School of Life Sciences, Bengbu Medical College, Bengbu, China.

${ }^{6}$ Department of Clinical Laboratory, The First Affiliated Hospital of Bengbu Medical College, Bengbu, China.

\section{References}

1. Sung H, Ferlay J, Siegel RL, Laversanne M, Soerjomataram I, Jemal A, et al. Global Cancer Statistics 2020: GLOBOCAN Estimates of Incidence and Mortality Worldwide for 36 Cancers in 185 Countries. CA Cancer J Clin. 2021;71:209-49.

2. Taieb J, André T, Auclin E. Refining adjuvant therapy for non-metastatic colon cancer, new standards and perspectives. Cancer Treat Rev. 2019;75:1-11.

3. Vogel G. Malaria as lifesaving therapy. Science. 2013;342:686.

4. Qin L, Chen C, Chen L, Xue R, Ou-Yang M, Zhou C, et al. Worldwide malaria incidence and cancer mortality are inversely associated. Infect Agent Cancer. 2017;12:14.

5. Chen L, He Z, Qin L, Li Q, Shi X, Zhao S, et al. Antitumor effect of malaria parasite infection in a murine Lewis lung cancer model through induction of innate and adaptive immunity. PLoS One. 2011;6:e24407.

6. Liu Q, Yang Y, Tan X, Tao Z, Adah D, Yu S, et al. Plasmodium parasite as an effective hepatocellular carcinoma antigen glypican-3 delivery vector. Oncotarget. 2017;8:24785-96. 
7. Tong ZZ, Fang ZM, Zhang Q, Zhan Y, Zhang Y, Jiang WF, et al. Plasmodium yoelii infection inhibits murine leukaemia WEHI-3 cell proliferation in vivo by promoting immune responses. Infect Dis Poverty. 2018;7:48.

8. Ji-Chen Q, Hui Z, Yu-Meng J, Yu-Ting Y, Jia-Jun D, Zheng-Zheng W, et al. [Anti-tumor effect of infection on melanoma in mice]. Chinese Journal of Schistosomiasis Control. 2017;29:315-9.

9. Adah D, Yang Y, Liu Q, Gadidasu K, Tao Z, Yu S, et al. Plasmodium infection inhibits the expansion and activation of MDSCs and Tregs in the tumor microenvironment in a murine Lewis lung cancer model. Cell Commun Signal. 2019;17:32.

10. Qin L, Zhong M, Adah D, Qin L, Chen X, Ma C, et al. A novel tumour suppressor IncRNA F630028010Rik inhibits lung cancer angiogenesis by regulating miR-223-3p. J Cell Mol Med. 2020;24:3549-59.

11. Macheret M, Halazonetis TD. DNA replication stress as a hallmark of cancer. Annu Rev Pathol. 2015;10:425-48.

12. Bessou M, Lopez J, Gadet R, Deygas M, Popgeorgiev N, Poncet D, et al. The apoptosis inhibitor Bcl-xL controls breast cancer cell migration through mitochondria-dependent reactive oxygen species production. Oncogene. 2020;39:3056-74.

13. Fulda S. Exploiting mitochondrial apoptosis for the treatment of cancer. Mitochondrion. 2010;10:598-603.

14. Yamaguchi R, Lartigue L, Perkins G. Targeting $\mathrm{Mcl}-1$ and other $\mathrm{Bcl}-2$ family member proteins in cancer therapy. Pharmacol Ther. 2019;195:13-20.

15. Kim S-G, Seo S-H, Shin J-H, Yang J-P, Lee SH, Shin E-H. Increase in the nuclear localization of PTEN by the Toxoplasma GRA16 protein and subsequent induction of p53-dependent apoptosis and anticancer effect. J Cell Mol Med. 2019;23:3234-45.

16. Xie L-L, Shi F, Tan Z, Li Y, Bode AM, Cao Y. Mitochondrial network structure homeostasis and cell death. Cancer Sci. 2018;109:3686-94.

17. Suliman HB, Piantadosi CA. Mitochondrial Quality Control as a Therapeutic Target. Pharmacol Rev. 2016;68:20-48.

18. George J, Ahmad N. Mitochondrial Sirtuins in Cancer: Emerging Roles and Therapeutic Potential. Cancer Res. 2016;76:2500-6.

19. Ng MYW, Wai T, Simonsen A. Quality control of the mitochondrion. Dev Cell. 2021;56:881-905.

20. Zhu J, Wang KZQ, Chu CT. After the banquet: mitochondrial biogenesis, mitophagy, and cell survival. Autophagy. 2013;9:1663-76.

21. Palikaras K, Tavernarakis N. Mitochondrial homeostasis: the interplay between mitophagy and mitochondrial biogenesis. Exp Gerontol. 2014;56:182-8.

22. Romero-Garcia S, Prado-Garcia H, Valencia-Camargo AD, Alvarez-Pulido A. Lactic Acidosis Promotes Mitochondrial Biogenesis in Lung Adenocarcinoma Cells, Supporting Proliferation Under Normoxia or Survival Under Hypoxia. Front Oncol. 2019;9:1053. 
23. Bernardini JP, Lazarou M, Dewson G. Parkin and mitophagy in cancer. Oncogene. 2017;36:1315-27.

24. Wang Y, Liu H-H, Cao Y-T, Zhang L-L, Huang F, Yi C. The Role of Mitochondrial Dynamics and Mitophagy in Carcinogenesis, Metastasis and Therapy. Front Cell Dev Biol. 2020;8:413.

25. Onishi M, Yamano K, Sato M, Matsuda N, Okamoto K. Molecular mechanisms and physiological functions of mitophagy. EMBO J. 2021;40:e104705.

26. Avetisyan A, Salzberg A. Accurate elimination of superfluous attachment cells is critical for the construction of functional multicellular proprioceptors in Drosophila. Cell Death Differ. 2019;26:1895-904.

27. Vera-Ramirez L, Vodnala SK, Nini R, Hunter KW, Green JE. Autophagy promotes the survival of dormant breast cancer cells and metastatic tumour recurrence. Nat Commun. 2018;9:1944.

28. Kamino H, Nakamura Y, Tsuneki M, Sano H, Miyamoto Y, Kitamura N, et al. Mieap-regulated mitochondrial quality control is frequently inactivated in human colorectal cancer. Oncogenesis. 2016;4:e181.

29. Maddalena F, Sisinni L, Lettini G, Condelli V, Matassa DS, Piscazzi A, et al. Resistance to paclitxel in breast carcinoma cells requires a quality control of mitochondrial antiapoptotic proteins by TRAP1. Mol Oncol. 2013;7:895-906.

30. Kawakubo M, Cunningham TJ, Demehri S, Manstein D. Fractional Laser Releases Tumor-Associated Antigens in Poorly Immunogenic Tumor and Induces Systemic Immunity. Sci Rep. 2017;7:12751.

31. Tanaka K. The PINK1-Parkin axis: An Overview. Neurosci Res. 2020;159:9-15.

32. Ruan H, Leibowitz BJ, Zhang L, Yu J. Immunogenic cell death in colon cancer prevention and therapy. Mol Carcinog. 2020;59:783-93.

33. Lannagan TR, Jackstadt R, Leedham SJ, Sansom OJ. Advances in colon cancer research: in vitro and animal models. Curr Opin Genet Dev. 2021;66:50-6.

34. Evan GI, Vousden KH. Proliferation, cell cycle and apoptosis in cancer. Nature. 2001;411:342-8.

35. Yang Q, Ni L, Imani S, Xiang Z, Hai R, Ding R, et al. Anlotinib Suppresses Colorectal Cancer Proliferation and Angiogenesis via Inhibition of AKT/ERK Signaling Cascade. Cancer Manag Res. 2020;12:4937-48.

36. Scholzen T, Gerdes J. The Ki-67 protein: from the known and the unknown. J Cell Physiol. 2000;182:311-22.

37. Hammarsten P, Josefsson A, Thysell E, Lundholm M, Hagglof C, Iglesias-Gato D, et al. Immunoreactivity for prostate specific antigen and Ki67 differentiates subgroups of prostate cancer related to outcome. Mod Pathol. 2019;32:1310-9.

38. Yang C, Zhang J, Ding M, Xu K, Li L, Mao L, et al. Ki67 targeted strategies for cancer therapy. Clin Transl Oncol. 2018;20:570-5.

39. Cheng X, Ferrell JE. Apoptosis propagates through the cytoplasm as trigger waves. Science. 2018;361:607-12. 
40. Mohammad RM, Muqbil I, Lowe L, Yedjou C, Hsu H-Y, Lin L-T, et al. Broad targeting of resistance to apoptosis in cancer. Semin Cancer Biol. 2015;35(Suppl):78-103.

41. Chen $S$, Wang $Y, X u M$, Zhang $L$, Su Y, Wang $B$, et al. miR-1184 regulates the proliferation and apoptosis of colon cancer cells via targeting CSNK2A1. Mol Cell Probes. 2020;53:101625.

42. Ke B, Tian M, Li J, Liu B, He G. Targeting Programmed Cell Death Using Small-Molecule Compounds to Improve Potential Cancer Therapy. Med Res Rev. 2016;36:983-1035.

43. Luna-Vargas MPA, Chipuk JE. The deadly landscape of pro-apoptotic BCL-2 proteins in the outer mitochondrial membrane. FEBS J. 2016;283:2676-89.

44. Aubrey BJ, Kelly GL, Janic A, Herold MJ, Strasser A. How does p53 induce apoptosis and how does this relate to p53-mediated tumour suppression? Cell Death Differ. 2018;25:104-13.

45. Lopez J, Tait SWG. Mitochondrial apoptosis: killing cancer using the enemy within. Br J Cancer. 2015;112:957-62.

46. Bock FJ, Tait SWG. Mitochondria as multifaceted regulators of cell death. Nat Rev Mol Cell Biol. 2020;21:85-100.

47. Czabotar PE, Lessene G, Strasser A, Adams JM. Control of apoptosis by the BCL-2 protein family: implications for physiology and therapy. Nature reviews Molecular cell biology. 2014;15:49-63.

48. Badrinath N, Yoo SY. Mitochondria in cancer: in the aspects of tumorigenesis and targeted therapy. Carcinogenesis. 2018;39:1419-30.

49. Sun D, Tao W, Zhang F, Shen W, Tan J, Li L, et al. Trifolirhizin induces autophagy-dependent apoptosis in colon cancer via AMPK/mTOR signaling. Signal Transduct Target Ther. 2020;5:174.

50. He W, Tao W, Zhang F, Jie Q, He Y, Zhu W, et al. Lobetyolin induces apoptosis of colon cancer cells by inhibiting glutamine metabolism. J Cell Mol Med. 2020;24:3359-69.

51. Momcilovic M, Jones A, Bailey ST, Waldmann CM, Li R, Lee JT, et al. In vivo imaging of mitochondrial membrane potential in non-small-cell lung cancer. Nature. 2019;575:380-4.

52. Chiu Y-H, Lin S-CA, Kuo C-H, Li C-J. Molecular Machinery and Pathophysiology of Mitochondrial Dynamics. Front Cell Dev Biol. 2021;9:743892.

53. Porporato PE, Filigheddu N, Pedro JMB-S, Kroemer G, Galluzzi L. Mitochondrial metabolism and cancer. Cell Res. 2018;28:265-80.

54. Liu L, Li Y, Wang J, Zhang D, Wu H, Li W, et al. Mitophagy receptor FUNDC1 is regulated by PGC1a/NRF1 to fine tune mitochondrial homeostasis. EMBO Rep. 2021;22:e50629.

55. Scarpulla RC. Nucleus-encoded regulators of mitochondrial function: integration of respiratory chain expression, nutrient sensing and metabolic stress. Biochim Biophys Acta. 2012;1819:1088-97.

56. Yang H, Yang R, Liu H, Ren Z, Wang C, Li D, et al. Knockdown of peroxisome proliferator-activated receptor gamma coactivator-1 alpha increased apoptosis of human endometrial cancer HEC-1A cells. Onco Targets Ther. 2016;9:5329-38.

57. Tang J, Lu L, Liu Y, Ma J, Yang L, Li L, et al. Quercetin improve ischemia/reperfusion-induced cardiomyocyte apoptosis in vitro and in vivo study via SIRT1/PGC-1 a signaling. J Cell Biochem. 
2019;120:9747-57.

58. Shao C-S, Zhou X-H, Miao Y-H, Wang P, Zhang Q-Q, Huang Q. In situ observation of mitochondrial biogenesis as the early event of apoptosis. iScience. 2021;24:103038.

59. Kleih M, Böpple K, Dong M, Gaißler A, Heine S, Olayioye MA, et al. Direct impact of cisplatin on mitochondria induces ROS production that dictates cell fate of ovarian cancer cells. Cell Death Dis. 2019;10:851.

60. Kushwaha PP, Singh AK, Prajapati KS, Shuaib M, Fayez S, Bringmann G, et al. Induction of apoptosis in breast cancer cells by naphthylisoquinoline alkaloids. Toxicol Appl Pharmacol. 2020;409:115297.

61. LeBleu VS, O'Connell JT, Gonzalez Herrera KN, Wikman H, Pantel K, Haigis MC, et al. PGC-1a mediates mitochondrial biogenesis and oxidative phosphorylation in cancer cells to promote metastasis. Nat Cell Biol. 2014;16:992-1003.

62. Parzych KR, Klionsky DJ. An overview of autophagy: morphology, mechanism, and regulation. Antioxid Redox Signal. 2014;20:460-73.

63. Settembre C, Fraldi A, Medina DL, Ballabio A. Signals from the lysosome: a control centre for cellular clearance and energy metabolism. Nat Rev Mol Cell Biology. 2013;14:283-96.

64. Li Y, Zhou D, Ren Y, Zhang Z, Guo X, Ma M, et al. Mir223 restrains autophagy and promotes CNS inflammation by targeting ATG16L1. Autophagy. 2019;15:478-92.

65. Ilyas G, Zhao E, Liu K, Lin Y, Tesfa L, Tanaka KE, et al. Macrophage autophagy limits acute toxic liver injury in mice through down regulation of interleukin-1 $\beta$. J Hepatol. 2016;64:118-27.

66. Naik PP, Mukhopadhyay S, Panda PK, Sinha N, Das CK, Mishra R, et al. Autophagy regulates cisplatin-induced stemness and chemoresistance via the upregulation of CD44, ABCB1 and ADAM17 in oral squamous cell carcinoma. Cell Prolif. 2018;51:e12411.

67. Yan C, Gong L, Chen L, Xu M, Abou-Hamdan H, Tang M, et al. PHB2 (prohibitin 2) promotes PINK1PRKN/Parkin-dependent mitophagy by the PARL-PGAM5-PINK1 axis. Autophagy. 2020;16:419-34.

68. Ashrafi G, Schwarz TL. The pathways of mitophagy for quality control and clearance of mitochondria. Cell Death Differ. 2013;20:31-42.

69. Li Q, Qi F, Meng X, Zhu C, Gao Y. Mst1 regulates colorectal cancer stress response via inhibiting Bnip3-related mitophagy by activation of JNK/p53 pathway. Cell Biol Toxicol. 2018;34:263-77.

70. Cui Z, Liu L, Kwame Amevor F, Zhu Q, Wang Y, Li D, et al. High Expression of miR-204 in Chicken Atrophic Ovaries Promotes Granulosa Cell Apoptosis and Inhibits Autophagy. Front Cell Dev Biol. 2020;8:580072.

\section{Figures}

\section{Figure 1}


$P$. yoelii infection suppressed tumor growth in a murine colon cancer model. Balb/c mice were subcutaneously injected CT26.WT mice colon cancer cells under the right forearm. On the sixth day, the tumor-bearing mice were intraperitoneally injected with either $P$. yoelii-infected erythrocytes or uninfected erythrocytes. (a) Tumor volume was measured over time from the date of tumor formation. Day15 (t-test, $t_{(8)}=10.01, P<0.0001$ ); Day18 (t-test, $t_{(8)}=6.515, P=0.0002$ ); Day21 (t-test, $t_{(8)}=6.290, P=0.0002$ ); Day24 (ttest, $t_{(8)}=6.290, P=0.0002$ ). The mice were sacrificed on day 24 , and tumors were harvested for weighing, photographing (b), and further analysis. (c) Weight of the Tumors mass (t-test, $t_{(8)}=6.618, P=0.0002$ ). CT26.WT denotes the control group and CT26.WT+P.y denotes the experimental group. The results are shown as mean \pm SEM $(\mathrm{n}=5) . \star \star \star \star P<0.001, * \star \star \star * P<0.0001$.

\section{Figure 2}

Plasmodium infection inhibited the proliferation of tumor cells in colon cancer tissues. Immunohistochemical staining (a) demonstrating that $P$. yoelii infection impacted colon cancer proliferation in mice $(\times 400)$. The percentage of Ki67 expression in tumor tissues of the two groups (t-test, $\left.t_{(8)}=17.51, P<0.0001\right)($ b). Brown areas represent positive expression. Scale bar $=50 \mu \mathrm{m}$. The results are shown as mean \pm SEM $(\mathrm{n}=5)$. ${ }^{\star * \star *} P<0.0001$.

\section{Figure 3}

Apoptosis in colon cancer induction by Plasmodium infection. (a) Apoptosis was observed under a fluorescence microscope $(\times 400)$. (b) Quantitative estimation of the proportion of apoptotic cells in each experimental group (t-test, $t_{(8)}=22.31, P<0.0001$ ). Green fluorescence indicates the nuclei of TUNELpositive cells, blue represents DAPI stained nuclei, and Merge indicates the two fluorescence superpositions. The results are shown as mean \pm SEM $(n=5)$. Scale bar $=50 \mu m$. ${ }^{\star \star \star \star} P<0.0001$.

\section{Figure 4}

The mitochondrial pathway regulation by Plasmodium infection in colon cancer-being mice. Western blot analysis (a) shown the expression of mitochondrial-mediated proteins (Bax, Bcl-2, Caspase-9, and Caspase-3). Quantification of Western blot signals from (b). Bax (t-test, $\left.t_{(8)}=9.438, P<0.0001\right)$; Bcl-2 (ttest, $t_{(8)}=3.822, P=0.0051$ ); Caspase-9 (t-test, $t_{(8)}=5.462, P=0.0006$ ); Caspase-3 (t-test, $t_{(8)}=3.836$, $P=0.0050)$; Cleaved Caspase-3 (t-test, $\left.t_{(8)}=11.36, P<0.0001\right)$. Immunohistochemistry Staining (c) and quantification of apoptosis protein expression (d) in the mitochondrial pathway. Bax (t-test, $t_{(8)}=7.222$, $P<0.0001$ ); Bcl-2 (t-test, $\left.t_{(8)}=10.92, P<0.0001\right)$; Caspase-9 (t-test, $t_{(8)}=7.465, P<0.0001$ ); Caspase-3 (t-test, 
$\left.t_{(8)}=10.23, P<0.0001\right)$; Cleaved Caspase-3 (t-test, $\left.t_{(8)}=7.458, P<0.0001\right)$. The results are presented as mean

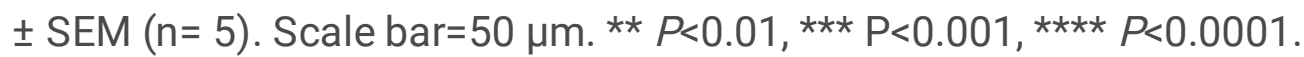

\section{Figure 5}

Changes of tumor cells ultrastructure after Plasmodium infection. The ultrastructural changes of tumor cells in the two groups were observed under TEM (a) (magnification×2000) (b) (magnification×6000). The chromatin of the nucleus was massive agglutination and the organelles were severely swollen in CT26.WT+P.y group. The mitochondria ( $M$, red arrow) were swollen, the cristae disappeared and vacuolated, and the chromatin clumpy agglutination and nuclear disintegration ( $\mathrm{N}$ : nucleus). The number of autolysosomes (ASS, blue arrow) in the CT26.WT+P.y group was more than that in the CT26.WT group. (a) Scale bar $=5 \mu \mathrm{m}$. (b) Scale bar $=1 \mu \mathrm{m}$.

\section{Figure 6}

Plasmodium infection inhibited mitochondrial biogenesis in colon cancer. Western blot analysis (a) showed the expression of PGC-1a protein was decreased by Plasmodium-treated colon cancer tissues. GAPDH was detected as a loading control. The density ratio of PGC-1a/ GAPDH is shown on the right (b).

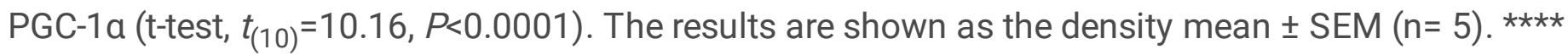
$P<0.0001$.

\section{Figure 7}

Plasmodium infection inhibited mitophagy in the murine colon cancer model. The expression of proteins associated with PINK1/Parkin pathway were inhibited after $P$. yoelii infection via Western blot analysis (a). Quantification of proteins expression normalized to GAPDH (b). PINK1 (t-test, $t_{(10)}=7.340, P<0.0001$ ); Parkin (t-test, $\left.t_{(10)}=4.829, P=0.0007\right)$. P. yoelii infection abated the levels of PINK1/Parkin. The results are shown as the density mean \pm SEM $(\mathrm{n}=5) .{ }^{*} P<0.01, * \star \star P<0.001$.

\section{Figure 8}

The anti-colon cancer mechanisms of Plasmodium infection in vivo. In the murine colon cancer model, Plasmodium infection inhibited the proliferation of tumor cells and induced mitochondria-mediated apoptosis. Furthermore, Plasmodium infection disturbed mitochondrial biogenesis leading to proliferation inhibition and apoptosis promotion. Mitophagy was attenuated by Plasmodium infection 
contributing to mitochondrial dysfunction. Plasmodium infection inhibited the growth of tumors to exert the tumor suppression function.

\section{Supplementary Files}

This is a list of supplementary files associated with this preprint. Click to download.

- Graphicalabstract.tif 